\section{Case management}

DeAR SirS

Dr Abrahamson alerts us to the numbers of mental health professionals germinating into case managers (Psychiatric Bulletin August 1993, 17, 499). This trend is matched by a large volume of literature about the subject which is reviewed by Thornicroft (1990).

Various models of case managements have been proposed but, in its most ambitious form, the job indeed requires a multitude of skills including engagement, assessment, consultation with families, psychoeducation, crisis intervention and advocacy (Kanter, 1989). The breadth and intensity of a psychiatrist's training, and the extent of his or her knowledge about the biological, social and psychological aspects of mental illness make psychiatrists and trainees particularly well suited to the role of case manager. Indeed, if psychiatrists do not participate in case management there is a danger that we will be reduced to medication prescribers alone.

Supervision is of paramount importance to the success of case management and is often poorly addressed by its exponents. Unsupervised and unsupported case managers are at risk of becoming demoralised by working with different clients, suffering emotional over-involvement and clinical isolation.

Having had personal experience of case management and read about the roles and functions of the case manager, we still wonder what are their responsibilities, and to whom are they accountable? Will the advent of case management mean that the other remaining function of psychiatrists is to carry the legal can, despite our increasingly peripheral involvement, when the patient's management goes wrong? To avoid these problems, and prevent our own marginalisation, consultant psychiatrists must surely continue to supervise the other case managers within the multidisciplinary team setting.

Several studies have suggested that case management improves the outcome for the chronic mentally ill (Solomon, 1992). Psychiatrists are indeed well advised to participate in the debates surrounding case management and its implementation. It may well make a useful contribution to the community based psychiatric care of the 1990s.

ROBERT Chaplin

Royal Park Hospital

Parkville Melbourne

Victoria 3052

Australia

\section{References}

KANTER, J. (1989) Clinical case management: definition, principles, components. Hospital and Community Psychiatry, 40, 361-368.
Solomon, P. (1992) The efficacy of case management services for severely mentally disabled clients. Community Mental Health Journal, 28, 163-180.

ThORNICROFT, G. (1990) Case managers for the mentally ill. Social Psychiatry and Psychiatric Epidemiology, 25, 141-143.

\section{Resuscitation skills of psychiatric trainees}

DeAR SIRS

In their article (Psychiatric Bulletin, August 1993, 17, 489-491) Kosky and Spearpoint highlight serious deficiencies in the resuscitation skills of psychiatric trainees-findings which are compatible with my own experiences in psychiatry.

Their results are unsurprising. Trainees are rarely called upon to perform resuscitation. Any resuscitation skills that they had on entering psychiatric training rapidly decline with lack of practice and are only maintained with regular rehearsal - a facility not available to most trainees.

The guidelines for the management of cardiac arrest are periodically revised. Without regular retraining it is unlikely that a trainee will remain up to date even if their basic life support skills are intact.

Adequate assistance from trained nursing staff is vital as a trainee managing a cardiac arrest is likely to be the only doctor present but most psychiatric nurses have done little general nursing and feel inadequately trained in assisting at resuscitation.

Basic life support skills are expected of any doctor. However, advanced resuscitation skills (defibrillation, intubation, gaining central venous access etc) can only be expected when regular training in and practice of such techniques is available. No psychiatric hospital that I have worked in has provided this. In three years as a trainee I have had only two brief refresher sessions in basic cardio-pulmonary resuscitation.

As Kosky and Spearpoint note, poor resuscitation skills are not restricted to psychiatrists. David \& Prior-Willeard (1993) examined 30 MRCP Part II candidates and found that 29 had inadequate basic life support skills and lack of knowledge of current guidelines. Nineteen of these candidates went on to pass the MRCP. The authors conclude that the time has come for regular, compulsory training in resuscitation.

Hospitals should decide what level of competence they expect in resuscitation and then provide appropriate regular training for both medical and nursing staff.

\section{The Elms Clinic}

J. D. D. LAIDLAW

Banbury, Oxfordshire 


\section{Reference}

DAvid, J. \& Prior-Willeard, P. F. S. (1993) Resuscitation skills of MRCP candidates. British Medical Journal, 306, 1578-1579.

\section{DeAr Sirs}

I was interested to read the article concerning resuscitation skills of psychiatric trainees by Kosky and Spearpoint (Psychiatric Bulletin, August 1993, 17, 489-491) and endorse their recommendation that all doctors should have refresher courses on cardiopulmonary resuscitation.

It would seem that psychiatric hospitals are running tremendous risks in the way they are equipped to deal with medical emergencies and are fortunate that this is not exposed in the mass media. The risks are not purely because of poorly trained doctors in the skills of resuscitation, but in the rapidly decreasing time psychiatric nurses spend on general hospital placements in their training, in the poverty of equipment required in a medical emergency and in the level of complacency surrounding the notion that psychiatric patients are medically fit and healthy.

I felt that the authors had set their sights far too high; certainly if I was at the scene of a cardiac arrest in a psychiatric hospital and was presented with a defibrillator and an array of anti-arrhythmic drugs I would require as much resuscitation as the patient.

As a registrar in general psychiatry I was involved in two nightmares. The first required the rapid administration of intravenous fluid; a venflon was finally located on the patient's ward, a giving set on another ward, an out-of-date bag of saline in the extra care area at the other end of the hospital and no drip stand to be found. On another occasion a patient arrested following an overdose; she was maintained with cardiopulmonary resuscitation until an ambulance arrived. However, the ambulancemen somehow assumed that the patient could be transported down a large flight of stairs, through a long corridor and down another flight of stairs in a wheelchair, when she had no cardiac output. When they returned with an appropriate stretcher one of the ambulancemen himself was suffering from a severe asthmatic attack.

Following these traumatic experiences I have learnt the cardinal rules concerning medical emergencies in psychiatric hospitals.

(a) The patient needs to be removed from a psychiatric hospital as rapidly as possible. The first thing to do before initiating any procedures is to ensure that an ambulance is on the way and is aware that it is not a call to transfer routinely a patient from one hospital to another but that there is a real emergency.

(b) Anything other than first aid is unrealistic and dangerous. For example, to give drugs during an arrest assumes that there will be a competent nurse present to continue the cardiopulmonary resuscitation.

(c) Put in writing to the management your unease about the risks being taken.

(d) Hope that it is your colleagues who are on call when the next disaster occurs.

Alastair Neale

Young People's Centre

Mount Gould Hospital

Plymouth PLA 7QD

\section{Thyroid microsomal antibody}

DeAR SIRS

The paper by Suresh \& Robertson (Psychiatric Bulletin, August 1993, 17, 477-478) prompts me to direct attention to thyroid microsomal antibody (TMA) concentrations, an additional component of thyroid dysfunction in the population with Down's syndrome (Kohen \& Wise, 1992).

In this study of 30 randomly selected clinically euthyroid Down's syndrome subjects, TMA concentration results showed that $40 \%(n=12)$ had positive TMA (titre $>40$ ), of whom nine were aged under 50 and three over. Of the individuals negative for TMA, five were under 50 and 13 over $(P=0.03)$. The study concluded that this different of circulating TMA in subjects with Down's syndrome over 50 years of age could be the result of selective mortality of the younger group with positive TMA results.

Investigations of the high prevalence of thyroid dysfunction in Down's syndrome has brought great improvement to their wellbeing. The study of TMA concentration results may also bring new insight into the high rates of coronary heart disease, vascular and immunological disorders and Alzheimer's disease in this population. I believe that regular screening for thyroid dysfunction should be supplemented by TMA results to clarify this possible interaction between thyroid, immunology and pathology in the other systems of the Down's syndrome population.

Charing Cross Hospital

DORA KOHEN

London W6 8RF

\section{Reference}

KoHEN, D. \& Wise, P. (1992) Autoantibodies in Down's syndrome. Lancet, $340,430$.

\section{Second medical recommendations}

\section{DeAr Sirs}

I was interested to read the article on second medical recommendations for the Mental Health Act (Ung, Psychiatric Bulletin, August 1993, 17, 466-468). This highlighted, particularly in the case of general practitioners, the lack of independence in these assessments. 